\title{
An investigation into multi-domain simulation for a pantograph- catenary system
}

\author{
Pawet Zdziebko, ${ }^{1, *}$, Adam Martowicz ${ }^{1}$, and Tadeusz $\mathrm{Uhl}^{1}$ \\ ${ }^{1}$ AGH University of Science and Technology, Department of Robotics and Mechatronics, Al. A. Mickiewicza 30, 30-059 Krakow, \\ Poland
}

\begin{abstract}
There is a need for modelling various phenomena present in a pantograph-catenary structure, (e.g. wave propagation and its reflections, friction, aerodynamic and electromagnetic forces), which allows for a more reliable study on the dynamic behaviours of a railway pantograph, particularly in the case of high-speed trains. Hence, the creation a complex pantograph-catenary multi-domain model should help to effectively meet the above-mentioned requirements. The work presents a co-simulation approach to investigate the pantograph-catenary dynamic interaction. The elaborated co-simulation algorithm assumes data exchange between multibody models of a pantograph and finite element model of a catenary. The presented approach explores multi-domain phenomena that have an influence on the pantograph-catenary interaction. The nonlinear finite element catenary model takes into account the slackening of droppers, relatively large displacements and contact with the pantograph's slider, while the multibody model of the pantograph considers friction forces and suspension springs. Additionally, aerodynamic forces caused by wind acting on the pantograph were computed using the fluid structure interaction method and implemented in the dynamic simulation. The influence on the pantograph-catenary interaction caused by electromagnetic force acting on the pantograph was investigated, along with the influence of the locomotive's vertical vibrations and tilt.
\end{abstract}

\section{Introduction}

Pantograph - catenary system is used commonly in electrified railways. Its effective performance is necessary for maintaining high speeds during train travel. The contact force, as well as its variation, is one of the parameters which describes the quality of the pantograph-catenary interaction. It is measured between the pantograph's head and contact wire (Fig. 1) and can significantly fluctuate during a ride due to vibrations in this complex system. Therefore, contact force fluctuations may cause unexpected drops of the current flow efficiency and further power decreases. Numerical simulations have become an essential tool in the analysis of a pantograph-catenary system. Exact modelling is crucial for conducting proper analysis of the performance in this structure, especially at the design stage of its components.

For many years, pantograph-catenary dynamic interaction has been the interest of many authors. The newest models for simulating catenary structure are mainly based on the Finite Element Method (FEM) and
The Finite Difference Method (FDM) [1,2]. Finite element (FE) models allow one to consider the most important phenomena present in a catenary: wave propagation in the structure and nonlinearity (contacts, large displacements, slackening of droppers). In the case of the pantograph model, the newest publications [3], employ Multi Body Dynamics models (MB) because they allow them to consider a desired number of degrees of freedom (DOF), realistic kinematic behaviour and inertia properties of particular components.

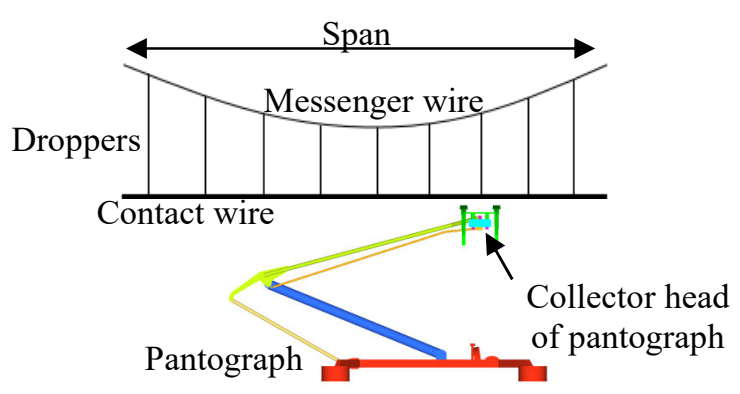

Fig. 1. Pantograph-catenary system.

\footnotetext{
*Corresponding author: zdziebko@agh.edu.pl
} 


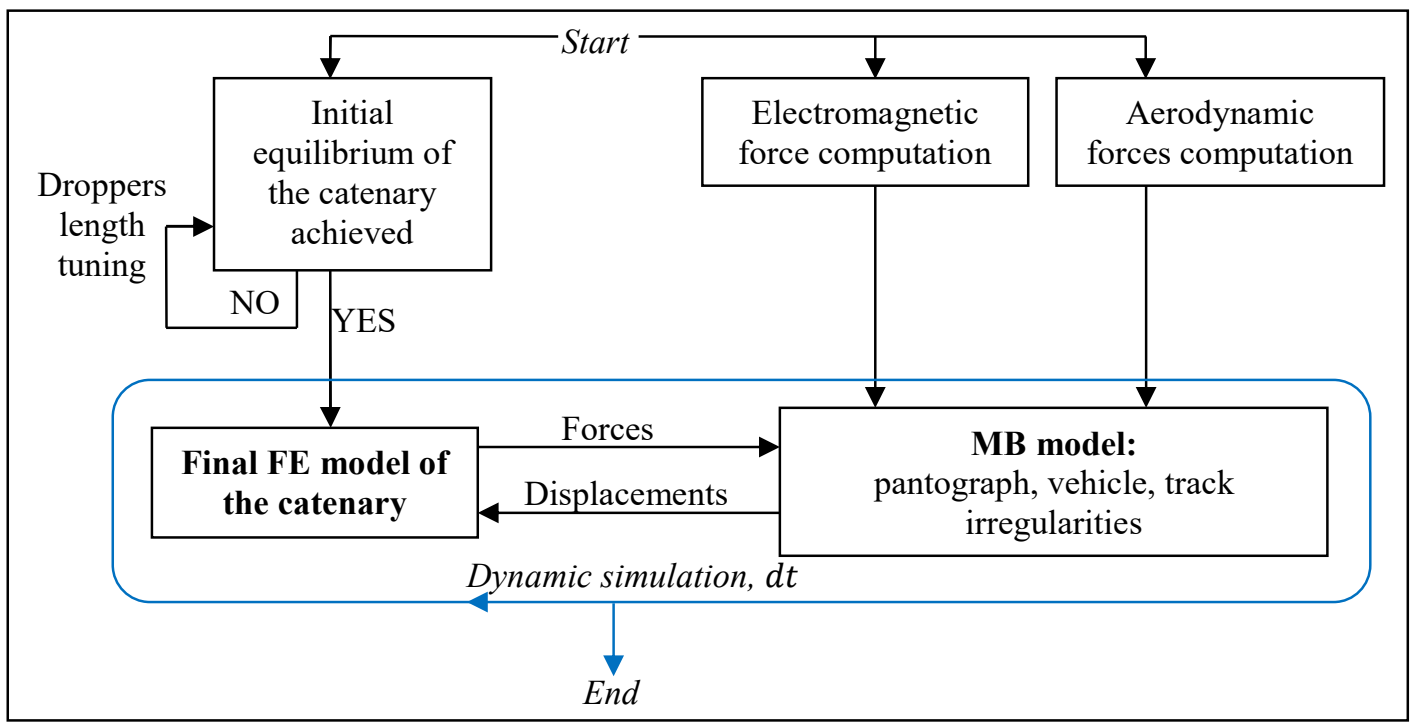

Fig. 2. Co-simulation algorithm.

The paper is organized as follows. After the present introductory in Section 1, Section 2 addresses the cosimulation algorithm proposed by the authors. The new approach considers multi-domain phenomena and it is an extension of the author's previous work [4]. Next, Section 3 describes the contributing models employed in the co-simulation. Subsequently, co-simulation results are presented in Section 4 and finally Section 5 concludes the paper.

\section{Co-simulation algorithm}

As mentioned before, the pantograph dynamic interaction with overhead lines (catenary) is disturbed by many different phenomena. The authors propose the cosimulation algorithm, which is presented in Fig 2. Firstly, three separate models are built:

- Initial FE model of a catenary - it is used to compute the final configuration of a catenary (with required pre-sag under gravity and tension of wires) by optimization of the droppers length.

- Model for electromagnetic force computation - this force acting on a collector strip is then passed into the MB model of a pantograph.

- Fluid structure interaction (FSI) model for aerodynamic forces computation - those forces acting on each component of a pantograph are passed into MB model.

Secondly, when final configuration of a catenary is achieved, the MB coupled track - rail vehicle pantograph model is established. Finally, the dynamic solution of pantograph - catenary interaction is computed with certain time step $d t$ in the co-simulation between FEM and MB codes. MB code is run first, and passes the displacement of the collector head (considering train speed and track excitations) into FEM code of a catenary. Subsequently, FEM code computes dynamic solution of a catenary under actual position of the collector head and passes the reaction forces acting on it into the MB code, where the MB model is calculated including forces delivered by FEM code. The procedure is repeated to cover the total simulation time [5].

\section{Multi-domain models}

\subsection{FEM catenary model}

The catenary structure employed in the analysis is similar to the one presented by the European Standard PN-EN 50318 [6]. It has a single contact wire and nine droppers per one span (which is $60 \mathrm{~m}$ long). The height of the traction catenary is $1.2 \mathrm{~m}$ and it has a stagger of $\pm 0.2 \mathrm{~m}$. For the pantograph-catenary dynamic interaction, ten identical spans were modelled (see Fig. 3.) Catenary wires are stretched by tensioning forces: 20 and $16 \mathrm{kN}$ respectively for contact and messenger wires. The messenger wire is fixed to the poles while the contact wire is constrained by registration arms mounted to the poles by prismatic joints. Poles are not modelled (adequate constrains are between 'space' and particular node). A precise description of employed catenary system can be found in ref [6].

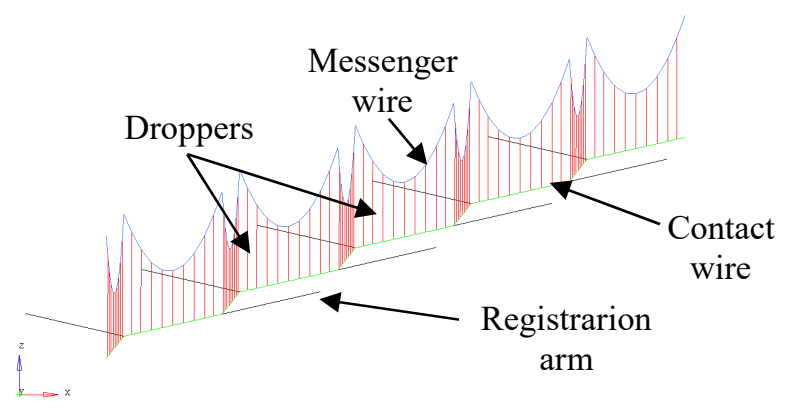

Fig. 3. Finite element model of the catenary.

FEM was employed for simulating the catenary system. The FE mesh was prepared in the Altair HyperMesh program, while the dynamic solution is computed in MSC.Marc solver (recommended for nonlinear models). The types of used FEs are listed in Table 1. 
Table 1. Types of elements in the FE model.

\begin{tabular}{|c|c|}
\hline Component & Element type \\
\hline Contact wire & \multirow{2}{*}{ Beam, el. 98} \\
\cline { 1 - 1 } Messenger wire & \\
\cline { 1 - 1 } Registration arms & \\
\cline { 1 - 1 } Droppers & Nonlinear spring \\
\hline
\end{tabular}

The employed beam elements (el. 98) include transverse shear effects. The detailed elements description can be found in [7]. In reality, droppers are made from steel cable and exhibit nonlinear characteristics. They can transfer tensioning load, but they are very compliant to compression (they do not transfer compressing loads). Therefore, the droppers are implemented in the model as nonlinear springs with the characteristics presented in Fig. 4.

\section{Stiffness of nonlinear droppers}

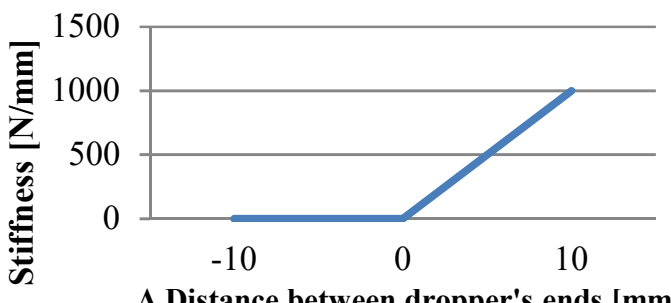

$\Delta$ Distance between dropper's ends [mm]

Fig. 4. Characteristics of nonlinear droppers.

Relatively large displacements are also considered in the FE model of the catenary. Dynamic transient solution of the proposed catenary model allows to consider wave propagation phenomena (and its reflections) in the structure. The wave propagation time was computed and it is $0.498 \mathrm{~s}$, therefore wave propagation velocity is equal to $433.7 \mathrm{~km} / \mathrm{h}$.

\subsection{Multibody rail vehicle-pantograph model}

The work concerns the pantograph mounted on the roof of a rail vehicle. The pantograph mechanism employed in this paper represents one which is commonly used in Europe - 160ECT (produced by EC Engineering). Basic kinematic diagram is presented in Fig. 5.

Dots in the image represent revolute joints (rotational axis is perpendicular to $\mathrm{ZX}$ plane), while triangle symbols represent montage points at the frame. Kinematic pairs in the pantograph mechanism are described in Table 2.

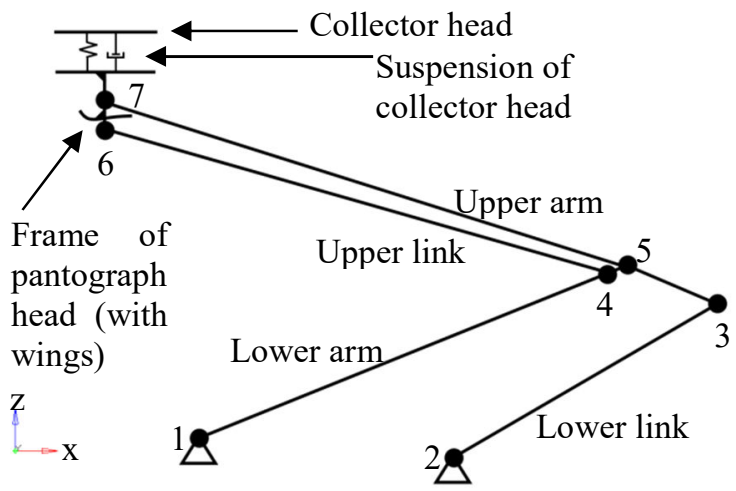

Fig. 5. Kinematic model of pantograph used in MB simulation.

Table 2. Revolute joints in pantograph.

\begin{tabular}{|c|c|c|}
\hline No. & Body 1 & Body 2 \\
\hline 1 & Frame & Lower arm \\
\hline 2 & Frame & Lower link \\
\hline 3 & Lower link & Upper arm \\
\hline 4 & Lower arm & Upper link \\
\hline 5 & Lower arm & Upper arm \\
\hline 6 & Upper link & Frame of pantograph head \\
\hline 7 & Upper arm & Frame of pantograph head \\
\hline
\end{tabular}

Fig. 6. Rail vehicle-pantograph MB model. 
Friction coefficient was introduced into revolute joint no. 1, that total friction force in pantograph is compliant with the norm PN-EN 50206 [8].

The Authors propose to take into consideration vibrations and tilt of a car body on which the pantograph is placed. Those kinematic constrains can significantly disturb the pantograph- catenary dynamic interaction. Utilized model of a rail vehicle assumes 6 DOF of a car body, see Fig 6. Therefore, it allows us to take into account the most important directions of pantograph excitation from car body (vertical, lateral vibrations and tilt). The model of the rail vehicle was built according to [9]. The parameters of the two-level suspension system in the model are shown in Table 3.

Table 3. Parameters of rail vehicle suspension system.

\begin{tabular}{|l|l|l|l|}
\hline Symbol & Description & Unit & Value \\
\hline $\mathrm{k}_{1 \mathrm{x}}$ & $\begin{array}{l}\text { Longitudinal stiffness of } \\
\text { primary suspension }\end{array}$ & $\mathrm{N} / \mathrm{mm}$ & 2667 \\
\hline $\mathrm{k}_{1 \mathrm{y}}$ & $\begin{array}{l}\text { Lateral stiffness of } \\
\text { primary suspension }\end{array}$ & $\mathrm{N} / \mathrm{mm}$ & 3925 \\
\hline $\mathrm{k}_{1 \mathrm{z}}$ & $\begin{array}{l}\text { Vertical stiffness of } \\
\text { primary suspension }\end{array}$ & $\mathrm{N} / \mathrm{mm}$ & 880 \\
\hline $\mathrm{c}_{1 \mathrm{x}}$ & $\begin{array}{l}\text { Longitudinal damping of } \\
\text { primary suspension }\end{array}$ & $\mathrm{Ns} / \mathrm{mm}$ & 0 \\
\hline $\mathrm{c}_{1 \mathrm{y}}$ & $\begin{array}{l}\text { Lateral damping of } \\
\text { primary suspension }\end{array}$ & $\mathrm{Ns} / \mathrm{mm}$ & 0 \\
\hline $\mathrm{c}_{1 \mathrm{z}}$ & $\begin{array}{l}\text { Vertical damping of } \\
\text { primary suspension }\end{array}$ & $\mathrm{Ns} / \mathrm{mm}$ & 170 \\
\hline $\mathrm{k}_{2 \mathrm{x}}$ & $\begin{array}{l}\text { Longitudinal stiffness of } \\
\text { secondary suspension }\end{array}$ & $\mathrm{N} / \mathrm{mm}$ & 20 \\
\hline $\mathrm{k}_{2 \mathrm{y}}$ & $\begin{array}{l}\text { Lateral stiffness of } \\
\text { secondary suspension }\end{array}$ & $\mathrm{N} / \mathrm{mm}$ & 476 \\
\hline $\mathrm{k}_{2 \mathrm{z}}$ & $\begin{array}{l}\text { Vertical stiffness of } \\
\text { secondary suspension }\end{array}$ & $\mathrm{N} / \mathrm{mm}$ & 828 \\
\hline $\mathrm{c}_{2 \mathrm{x}}$ & $\begin{array}{l}\text { Longitudinal damping of } \\
\text { secondary suspension }\end{array}$ & $\mathrm{Ns} / \mathrm{mm}$ & 0.5 \\
\hline $\mathrm{c}_{2 \mathrm{y}}$ & $\begin{array}{l}\text { Lateral damping of } \\
\text { secondary suspension }\end{array}$ & $\mathrm{Ns} / \mathrm{mm}$ & 80 \\
\hline $\mathrm{c}_{2 \mathrm{z}}$ & $\begin{array}{l}\text { Vertical damping of } \\
\text { secondary suspension }\end{array}$ & $\mathrm{Ns} / \mathrm{mm}$ & 53 \\
\hline
\end{tabular}

Other geometrical parameters as well as mass and inertial properties of the car body and bogies were taken according to the model from ref. [9]. Those values are consistent with the parameters of rail electric multiple units (EMU) utilized in Poland, therefore the authors consider that proposed rail vehicle model represents realistic constrains of a pantograph on high-speed trains.
The proposed model allows us to set the required course of displacement on the longitudinal direction of a train. However, in this paper, constant speed of $160 \mathrm{~km} / \mathrm{h}$ was set in all dynamic simulations.

Railway track irregularities are commonly considered to be an important source of vibration excitation in high speed train systems. Therefore rail irregularities on the vertical direction were taken into consideration in numerical analysis (independently for right and left rails, denoted as $Z_{\text {rail_2 }}(x)$ and $Z_{\text {rail_1 }}(x)$ in Fig 6, respectively). Moreover, each wheel is excited with regard to appropriate phase delay (resulting from the distance between them). Irregularities of a track are often considered as random variables and are described by power spectral density, which includes information of the amplitudes and wavelengths of a particular irregularity. In this paper, the authors used PSD formula describing track irregularities (eq. 1) according to ERRI B176 [10, 11].

$$
S_{A}(\lambda)=2 \pi \frac{A_{A} \cdot \Omega_{c}^{2}}{\left(\left(\frac{2 \pi}{\lambda}\right)^{2}+\Omega_{r}^{2}\right) \cdot\left(\left(\frac{2 \pi}{\lambda}\right)^{2}+\Omega_{c}^{2}\right)}
$$

where $\Omega_{c}=0.82 \mathrm{rad} / \mathrm{m}, \Omega_{r}=0.02 \mathrm{rad} / \mathrm{m}$ are cut-off frequencies and $\lambda$ is the wavelength in $[\mathrm{m}] . A_{A}$ in $[\mathrm{m} \cdot \mathrm{rad}]$ is the amplitude of track irregularities and value of $2.1 \cdot 10^{-7}$ is a guideline for good quality track, while the value of $6.1 \cdot 10^{-7}$ corresponds to low quality track. In this paper $A_{A}=4.1 \cdot 10^{-7} \mathrm{~m} \cdot \mathrm{rad}$ was used and is interpreted as for a middle quality track.

On the basis of selected power spectral density, waveforms of track irregularities were computed using an inverse discrete Fourier transform with random phases. Used waveforms for right $\left(\mathrm{Z}_{\text {rail } 1}(\mathrm{x})\right)$ and left $\left(\mathrm{Z}_{\text {rail_2}}(\mathrm{x})\right)$ rails are presented in Fig. 7.

In normal operational conditions, the tilt of a car body is not present (it is on a negligible level), because track irregularities have too small amplitudes to significantly cause undesirable tilt of a car body. In reality, abnormal situations also show up, e.g. track cross level error (the situation when one rail is not at the exact same level like the second one) or uneven load distribution inside a coach can cause additional tilting of a car body which can additionally disturb pantographcatenary dynamic interaction. Vibrations and tilt of pantograph's frame excited from a car body are presented in Fig. 8 and Fig. 9.

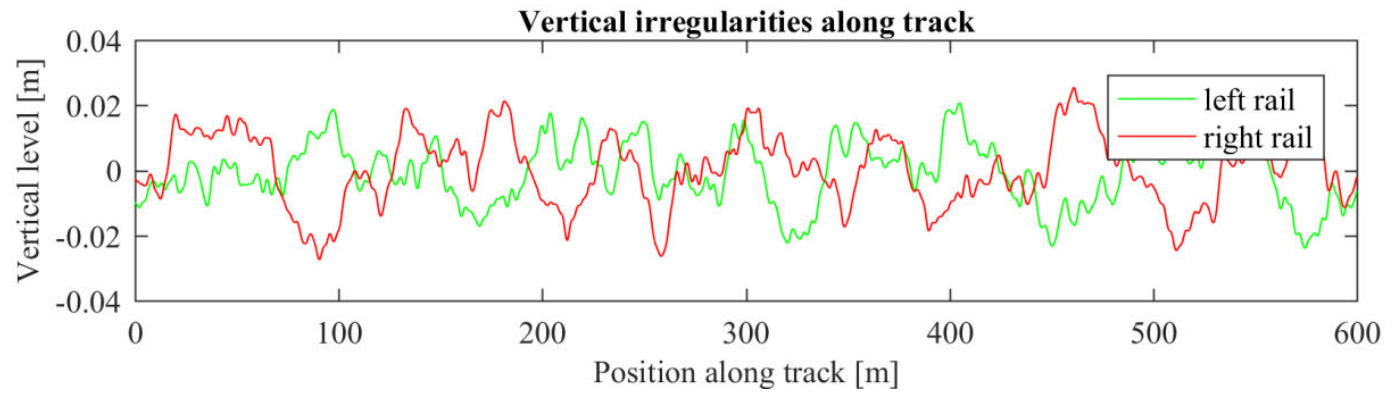

Fig. 7. Track irregularities for left and right rail. 


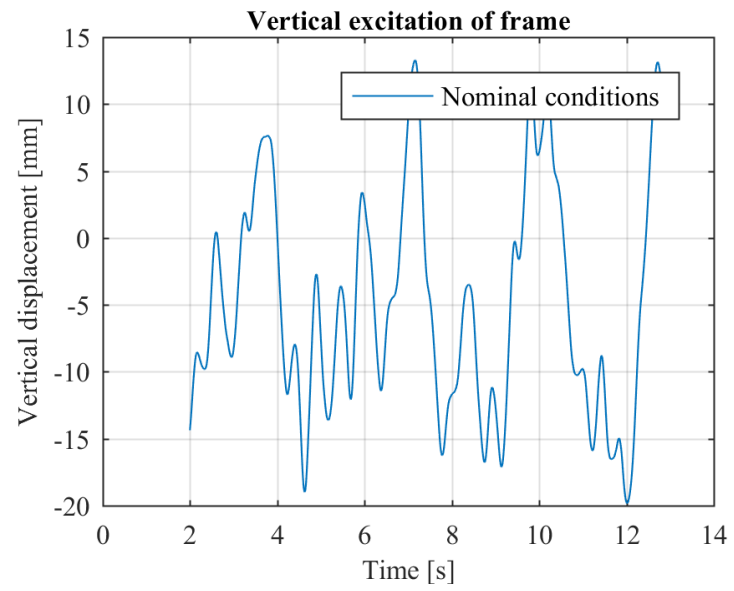

Fig. 8. Vertical displacement of pantograph's frame originating from car body vibrations.

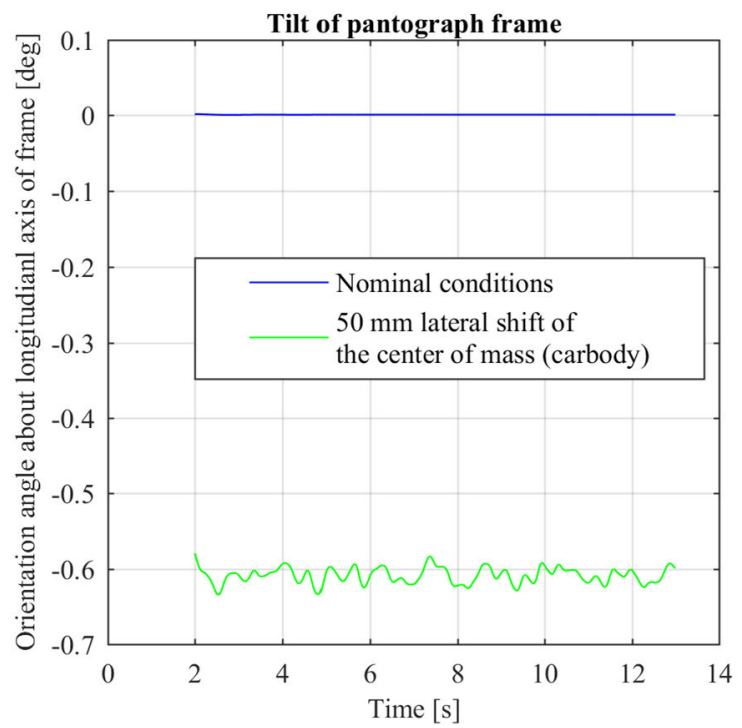

Fig. 9. Tilt of pantograph's frame originating from car body vibrations.

\subsection{Aerodynamics}

Wind flow is a source of large external forces acting on a pantograph mechanism in high-speed trains. Therefore, aerodynamic forces highly affect the nominal contact force (90-110N for DC systems according to [12]) between contact strips and contact wire. The static pressure exerted by a pantograph on a catenary wire should be kept within specific limits defined as functions of a train speed. On one hand, an increase of the pantograph static pressure makes the lifetime of contact strips shorter because of the high friction forces in that contact pair. On the other hand, at high-speed travel, the contact force shall be greater than for the normal operational condition to provide better contact at high speeds. The above-mentioned arguments confirm the need to take aerodynamic forces into account in pantograph-catenary dynamic simulations. In the paper, the authors prepared a numerical model of the pantograph for FSI analysis. Geometry of the pantograph was slightly simplified (small parts like screws and nuts were removed), but all important parts (frame, arms, links, pantograph head and wings) were modelled accurately.

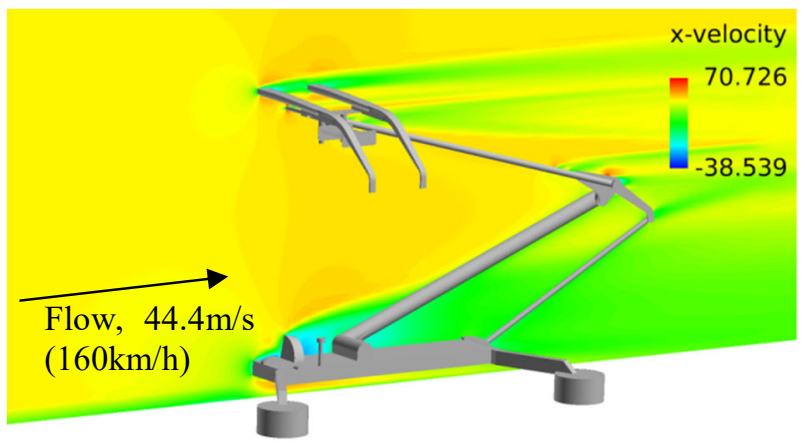

Fig. 10. FSI results: horizontal velocity in the middle plane.

The virtual wind tunnel has the following dimensions: $5 \times 5 \times 15 \mathrm{~m}$. The total number of elements in the model is 20300456. The model was computed in Altair AcuSolve solver, where a steady simulation strategy assumes Spalart-Allmaras turbulence model. In Fig. 10, velocity magnitude in the horizontal (longitudinal) direction is presented in the middle cross-section plane (legend is in $\mathrm{m} / \mathrm{s}$ ). Directly from the FSI analysis, drag, lift, and cross forces acting on each part of the pantograph were computed. Afterwards, those forces were introduced in the MB pantograph model and total uplift force, considering aerodynamic forces, was calculated. Finally, to validate the proposed FSI model, the obtained results were compared to those ones taken from the experiment in a real wind tunnel. The results of validation are presented in Table 4.

Table 4. Comparison of results from FSI model and experiment (flow speed: $160 \mathrm{~km} / \mathrm{h}$ ).

\begin{tabular}{|c|c|}
\hline \multicolumn{2}{|c|}{ Uplift force } \\
\hline Proposed model & $135.9 \mathrm{~N}$ \\
\hline Experiment & $136.4 \mathrm{~N}$ \\
\hline Error & $0.15 \%$ \\
\hline
\end{tabular}

The obtained results proof high accuracy of the proposed FSI model. Computed drag, lift and cross forces acting on components of the pantograph are considered to be within acceptable bounds.

\subsection{Electromagnetic force}

In normal operational conditions, a carbon strip mounted on the pantograph's collector head is in continuous contact with the contact wire, which carries current. In accordance with the principle of electromagnetic induction, a magnetic field is induced around the contact cable. The contact strip (through which the current also flows) is placed inside that magnetic field. Therefore, electromagnetic force is acting on the contact strip. This analysis aims to estimate that force. In the analysis, the magnetic field induced by three spans of a catenary were taken into consideration. The contact slider - contact wire schematic configuration is shown in Fig. 11, and it presents dimensions and currents in the 


\section{Pantograph-Catenary electromagnetic interface}

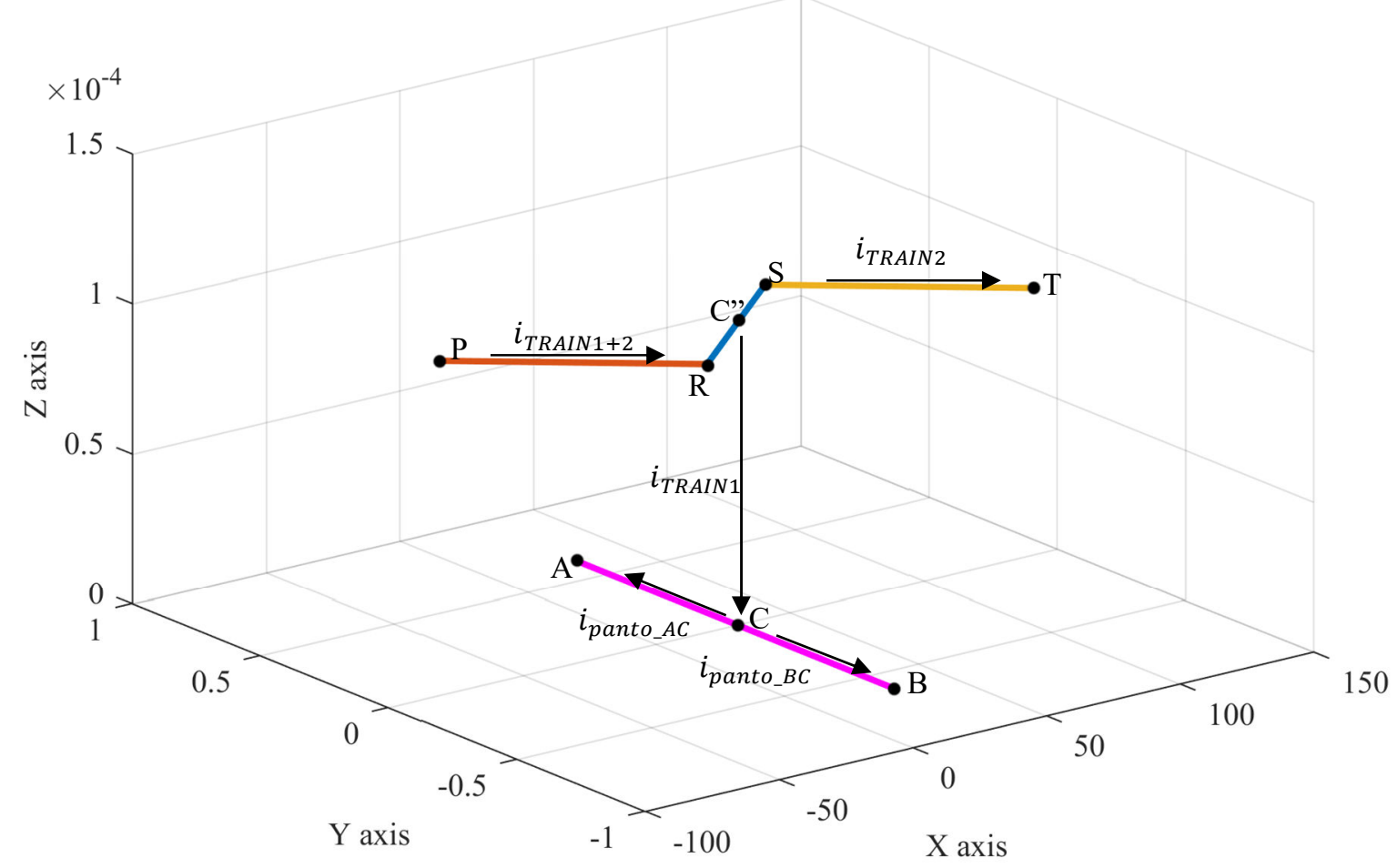

Fig. 11. Currents in electromagnetic pantograph-catenary interface. $X$ axis: position along track [m], $Y$ axis: dimensions of catenary stagger and width of contact strip [m], Z axis: distance between surfaces of contact wire collector strip [m] (global origin is located in the middle of contact strip). Different scales apply to the system coordinates.

employed structure. Section $|P R S T|$ is the contact wire while $|A B|$ is the contact strip. The utilized scenario of energy supply assumes two trains travelling one by one which are powered from the same traction current converter plant. According to the European Standard [13] in high-speed lines (3 kV DC system) train currents may be as high as $3500 \mathrm{~A}$ per one train. In the calculations the authors have taken following data:

$$
\begin{gathered}
i_{\text {TRAIN1 }}=i_{\text {TRAIN2 }}=3500[A] \\
i_{\text {TRAIN } 1+2}=7000[A] \\
i_{\text {PANTO_AC }}=\frac{i_{\text {TRAIN1 }}}{2}[A] \\
i_{\text {PANTO_BC }}=\frac{i_{\text {TRAIN1 }}}{2}[A]
\end{gathered}
$$

The distance between the slider strip and contact wire is to be $\overline{\left|C C^{\prime \prime}\right|}=0.0001 \mathrm{~m}$ and it represents irregularities in that interface [14]. The dimensions of the catenary spans correspond to the model presented in Section 3.1, while the length of the contact strip $\overline{|A B|}=1.2 \mathrm{~m}$. Magnetic induction at points on the contact strip is computed using the Biot-Savart's law. Segments of wires with current, which are taken into account in computing the magnetic field induction, are presented in Table 5.

The magnetic field in a particular point, e.g. B, can be computed using the Biot-Savart's rule. Let us first consider one finite length wire with current, e.g. $\left|R C^{\prime \prime}\right|$, see Fig. 12.
Table 5. Current in wires.

\begin{tabular}{|c|c|}
\hline Wire with current & Current [A] \\
\hline$|P R|$ & $i_{\text {TRAIN1+2 }}$ \\
\hline$\left|R C^{\prime \prime}\right|$ & $i_{\text {TRAIN1+2 }}$ \\
\hline$\left|C^{\prime \prime} S\right|$ & $i_{\text {TRAIN2 }}$ \\
\hline$|S T|$ & $i_{\text {TRAIN2 }}$ \\
\hline
\end{tabular}

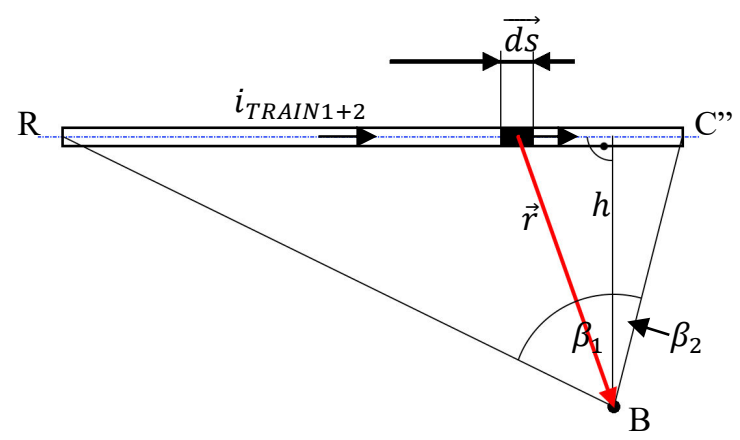

Fig. 12. Magnetic field from a finite length wire with current.

Magnetic induction at point B is (2):

$$
\overrightarrow{B_{R C}}=\frac{\mu_{0} \mu}{4 \pi} \int \frac{i_{T R A I N 1+2} \overrightarrow{d s} \times \vec{r}}{r^{3}}
$$


Where:

$\overrightarrow{B_{R C}}-$ magnetic field from wire $|R C "|$

$\mu_{0}, \mu$ - permeability of vacuum and relative permeability of air, respectively

$\overrightarrow{d s}-$ current length element in dir. of current

$\vec{r}$ - the full displacement vector from the wire element to the point at which the field is being computed

Solving the integral, we may find the magnitude of magnetic field at point $\mathrm{B}\left(h, \beta_{1}, \beta_{2}\right.$ are visible in Fig. 12):

$$
\left|\overrightarrow{B_{R C}}\right|=\frac{\mu_{0} \mu i_{T R A I N 1+2}}{4 \pi h}\left(\sin \beta_{1}+\sin \beta_{2}\right)
$$

The direction of $\overrightarrow{B_{R C}}$ is determined from the right hand rule. The magnetic field induced by other segments of wires is calculated in a similar way. The total magnetic field at point $B$ is

$$
\overrightarrow{B_{\text {Total_at_B }}}=\overrightarrow{B_{P R}}+\overrightarrow{B_{R C}}+\overrightarrow{B_{C " S}}+\overrightarrow{B_{S T}}
$$

The above procedure is repeated for all points at the contact strip $(|A B|$ is divided into $n$ segments of the length $d l$ - in the analysis $d l=0.1 \mathrm{~mm}$ ) and magnetic field at each segment $\overrightarrow{B_{\text {seg }}}$ is computed separately according to the procedure presented for point $B$.

The contact strip also carries current $\left(i_{\text {panto_AC }}\right.$ or $i_{\text {panto_BC }}$ depending on which side the actual segment is located), therefore at each segment $d l$, electromagnetic forces acting on it can be computed from (5)

$$
\overrightarrow{F_{\text {seg }}}=i_{\text {seg }} \overrightarrow{d l} \times \overrightarrow{B_{\text {seg }}}
$$

The total electromagnetic force acting on the collector strip is

$$
\overrightarrow{F_{\text {TOTAL }}}=\sum_{i=0}^{n} F_{i}{ }^{t h} \text { seg }
$$

Components in the global coordinate system of electromagnetic force which are computed for, and presented in the above scenario, equal

$$
\begin{gathered}
F_{\text {TOTAL_X }}=31.6 N, \quad F_{\text {TOTAL_Y }}=0 \mathrm{~N} \\
F_{\text {TOTAL_Z }}=-0.013 \mathrm{~N}
\end{gathered}
$$

The obtained results were implemented into the $\mathrm{MB}$ model. Electromagnetic forces with the largest longitudinal component slightly reduce the contact force between the collector head and contact wire. A graph representing its influence on the contact force for different working heights of the pantograph is presented in Fig 13. Vertical lines represent the lowest and highest working range of the pantograph (for Polish operational conditions). The highest influence of the electromagnetic field on the contact force is observed for working heights of between 1.5 to $1.8 \mathrm{~m}$ - in this range, electromagnetic force causes a reduction of the nominal pressure up to $2 \mathrm{~N}$.

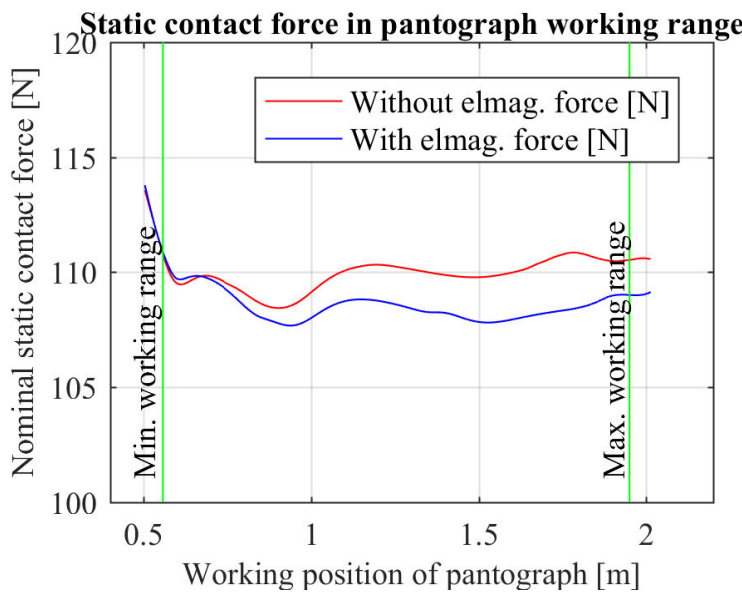

Fig. 13. Influence of electromagnetic force on actual static contact force in the pantograph working range.

\section{Co-simulation results}

The analysis of pantograph - catenary dynamic interaction was performed according to the proposed multi-domain co-simulation algorithm (Section 2). All important phenomena concerning this system were taken into account (precisely presented in Section 3). In the analysis, the static contact pressure exerted by the collector head on the contact wire was set to $100 \mathrm{~N}$ and train travel velocity was equal to $160 \mathrm{~km} / \mathrm{h}$. In order to assess the quality of the pantograph-catenary interaction, it is necessary to refer the results to reference values. According to Ambrosio et al. [15], the European Standard PN-EN 50367 describes important factors of the pantograph-catenary interaction quality (and their ranges of acceptance) by means of:

a. mean contact force: $F_{M}$

b. standard deviation of contact force: $\sigma<0.3 F_{M}$

c. max. contact force $F_{M A X}<350 N$

d. max. contact wire uplift at steady-arm

$$
C W_{u p} \leq 120 \mathrm{~mm}
$$

e. max. pantograph vertical amplitude

$$
\Delta_{Z} \leq 80 \mathrm{~mm}
$$

f. percentage of arcing NQ $\leq 0.2 \%$ The results received in proposed co-simulation procedure are presented in Fig. 14 and 15.

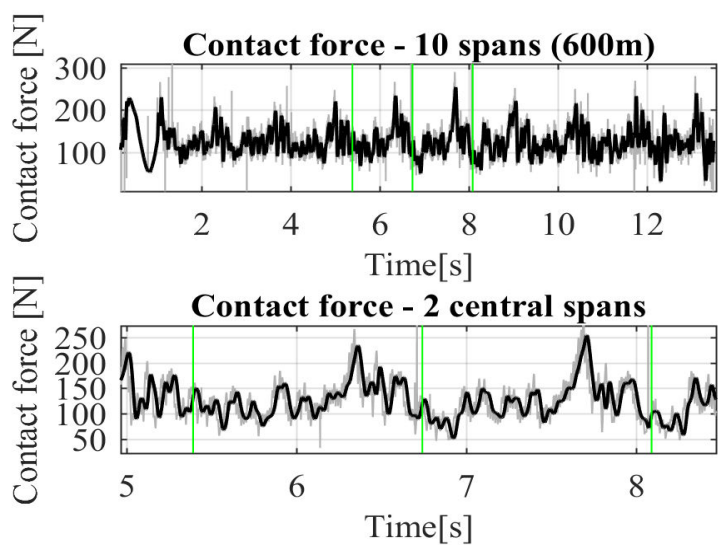

Fig. 14. Contact force in pantograph-catenary interface. Grey: registered, black: filtered, vertical lines - ends of central spans. 


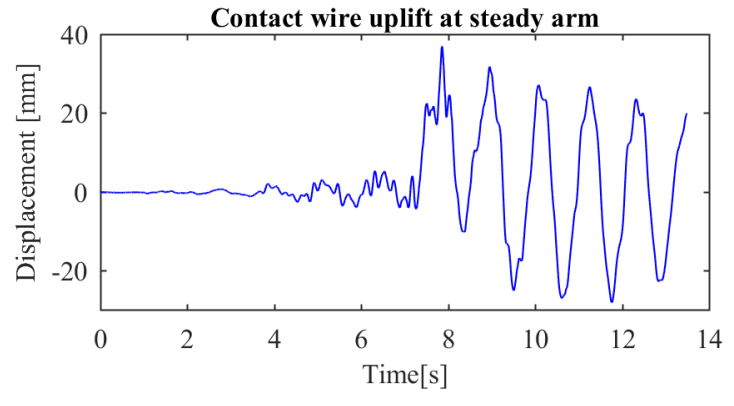

Fig.15. Contact wire uplift at steady arm between span no. 6 and 7 .

According to [6] statistical parameters from the simulation were computed for the segment of two central spans after filtration (low-pass filtering, cut-off frequency $20 \mathrm{~Hz}$ ). The results are presented in Table 6 . All parameters are within acceptance ranges.

Table 6. Results for pantograph - catenary interaction.

\begin{tabular}{|c|c|c|}
\hline Parameter & Simulation & $\begin{array}{c}\text { Allowed ranges } \\
{[15]}\end{array}$ \\
\hline $\begin{array}{c}\text { Mean contact force, } \\
F_{M}\end{array}$ & $126.4 \mathrm{~N}$ & $\begin{array}{c}108.4-134.8 \mathrm{~N} \\
\text { (for } \mathrm{v}=160 \mathrm{~km} / \mathrm{h})\end{array}$ \\
\hline $\begin{array}{c}\text { Standard deviation } \\
\text { of contact force, } \sigma\end{array}$ & $34.8 \mathrm{~N}$ & $\begin{array}{c}\sigma<0.3 F_{M} \\
\sigma<37.92 N \\
\left(\text { for achieved } F_{M}\right)\end{array}$ \\
\hline $\begin{array}{c}\text { Max. contact force, } \\
F_{M A X}\end{array}$ & $253.9 \mathrm{~N}$ & $F_{M A X}<350 \mathrm{~N}$ \\
\hline $\begin{array}{c}\text { Max. contact wire } \\
\text { uplift at steady-arm, } \\
C W_{u p}\end{array}$ & $37 \mathrm{~mm}$ & $C W_{u p} \leq 120 \mathrm{~mm}$ \\
\hline $\begin{array}{c}\text { Max. pantograph } \\
\text { vertical amplitude, } \\
\Delta_{Z}\end{array}$ & $50 \mathrm{~mm}$ & $\Delta_{Z} \leq 80 \mathrm{~mm}$ \\
\hline $\begin{array}{c}\text { Percentage of arcing } \\
\text { NQ* }\end{array}$ & 0 & $\mathrm{NQ} \leq 0.2 \%$ \\
\hline
\end{tabular}

*NQ is assumed to be a proportion of driving time, when the contact force is zero.

\section{Conclusions}

The main conclusions resulting from this paper are:

- In a pantograph-catenary interface, there are a number of different sources of disruption (vibrations of a contact wire and rail vehicle, aerodynamic and electromagnetic forces acting on a pantograph).

- Aerodynamic effects generate the highest external forces acting on a pantograph, which disturb its interaction with the catenary. FSI model was fully validated with the results from the wind tunnel experiment.

- 160ECT pantograph properly interacts with the catenary when the speed of train travel is equal to $160 \mathrm{~km} / \mathrm{h}$. The pantograph is expected to have performance accurately at lower speeds because aerodynamic effects are less significant.

- The proposed co-simulation algorithm allows us to consider the following important phenomena that have a non-negligible influence on a pantograph- catenary interaction. These are: wave propagation in cables, nonlinear droppers, large displacements, real kinematic chain of a pantograph, aerodynamic and electromagnetic forces acting on a pantograph and excitation from a rail vehicle.

- The presented algorithm might be used in the optimization of a pantograph-catenary interaction in virtual prototyping approach and that will be the subject of future work.

The work was supported by the AGH Grant no. 15.11.130.627 and carried out employing the infrastructure of the Centre of Energy AGH (Czarnowiejska 36, 30-054 Kraków, Poland).

\section{References}

1. S. Bruni, J. Ambrosio, A. Carnicero, Y.H. Cho, L. Finner, M. Ikeda, S.Y. Kwon, J.P. Massat, S. Stichel, M. Tur, W. Zhang, Veh. Sys. Dyn. 53, 412-435 (2015).

2. P. Zdziebko, T. Uhl, Arch. of Trans. 39, 77-85 (2016).

3. Y. Song, H. Ouyang, Z. Liu, G. Mei, H. Wang, X. Lu, Mech. and Mach. Theory 115, 35-59 (2017).

4. P. Zdziebko, A. Martowicz, T. Uhl, in: Projektowanie Mechatroniczne: zagadnienia wybrane (AGH, Kraków, 2017) (to be published) in Polish.

5. MSC.Adams Co-simulation Interface (2017).

6. PN-EN 50318 (2002).

7. MSC. Marc Element Library (2016).

8. PN-EN 50206:2010.

9. K. Zboinski, M. Dusza, Non. Dyn. 89, 863-885 (2017).

10. K. Karttunen, E. Kabo, A. Ekberg, Wear 314, 78-85 (2014).

11. P. Kurowski, A. Martowicz, T. Uhl, G. Lasko, Arch. of Trans. 23, 23-35 (2011).

12. PN-EN 50367:2012.

13. PN-EN 50388:2012.

14. Y. Zhao, G. Wu, G. Gao, W. Wang, L. Zhou, Jour. of the China Rail. Soc. 36, 28-32 (2014) in Chinese.

15. J. Ambrosio, J. Pombo, M. Pereira, Theo.\& App. Mech. Lett. 3 (2013). 